Revista Geográfica Digital. IGUNNE. Facultad de Humanidades. UNNE. Año 14. N²7.

Enero - Junio 2017. ISSN 1668-5180 Resistencia, Chaco

\title{
CUANTIFICACIÓN DE LA INCIDENCIA DEL ENSO EN LAS NEVADAS DE LOS ANDES ÁRIDOS DE SUDAMÉRICA EN EL PERIODO 1909-2015.
}

\section{QUANTIFICATION OF ENSO'S INCIDENCE IN THE SNOW OF THE ARID ANDES OF SOUTH AMERICA IN THE PERIOD 1909-2015.}

Dr. Poblete, Arnobio German. Profesor Titular. Instituto de Geografía Aplicada (IGA).UNSJ. Ayudante Alumna Atencio, Maira Antonella. UNSJ. Instituto de Geografía Aplicada (IGA) Prof. Vera, Maria Jose. Prof. Cátedra Climatología. Instituto de Geografía Aplicada (IGA). UNSJ.

\section{Resumen}

La población de Cuyo asocia el evento ENSO, con el monto de las nevadas en los Andes Áridos de manera directa, es decir, "Niño= Nevada", "Niña= No Nevada", teniendo en cuanta que de éstas depende el recurso hídrico que hace posible la existencia de los Oasis Cuyanos y las áreas agropecuarias de Chile Central.

Existen innumerables artículos que investigaron dicha asociación, sin embargo, son pocos los que se enfocan en la discriminación precisa de esa relación.

La conexión entre el ENSO y los derrames de los ríos cuyanos y chilenos fue tratada por diversos autores, pero en la mayoría de los casos abordaron su variabilidad sin cuantificar con precisión la incidencia de ese evento en los mismos.

Este trabajo, haciendo uso del índice Niño $3+4$, se propone cuantificar las aseveraciones respecto de dicha relación directa, en el período abril-septiembre, mediante herramientas estadísticas específicas y no paramétricas.

Palabras claves: ENSO- Incidencia- Nevadas- Andes Áridos- Sudamérica.

\begin{abstract}
The population of Cuyo associates the ENSO event, with the amount of snowfall in the Arid Andes directly, that is, "Niño = Snow", "Niña = No Snow ", taking into account that of these depends the water resource that Makes possible the existence of the Cuyanos Oasis and the agricultural areas of Central Chile.

There are innumerable articles that investigated this association, however, few are focused on the precise discrimination of that relationship.

The connection between ENSO and runoff of Cuyanos and Chilean rivers was treated by different authors, but in most cases they approached its variability without accurately quantifying the incidence of that event in them.

This work, using the Niño $3+4$ index, aims to quantify the assertions regarding this direct relationship, in the period April-September, using specific and non-parametric statistical tools.
\end{abstract}

Keywords: ENSO- Incidence- Snowfall- Arid Andes- South America.

Publicado en formato digital: Dr. Arnobio German Poblete, Ayudante Alumna Maira Antonella Atencio, y Prof. Maria José Vera. CUANTIFICACIÓN DE LA INCIENCIA DEL ENSO EN LAS NEVADAS DE LOS ANDES ÁRIDOS DE SUDAMÉRICA EN EL PERIODO 1909-2015. Revista Geográfica Digital. IGUNNE. Facultad de Humanidades. UNNE. Año 14. № 27 Enero - Junio 2017. ISSN 1668-5180 Resistencia, Chaco.

En: http://hum.unne.edu.ar/revistas/geoweb/default.htm 
Revista Geográfica Digital. IGUNNE. Facultad de Humanidades. UNNE. Año 14. №27.

Enero - Junio 2017. ISSN 1668-5180 Resistencia, Chaco

\section{Introducción}

Los medios periodísticos, académicos y público en general, asocian el evento ENSO (El Niño Southern Oscillation) con el monto de las nevadas en los Andes Áridos de manera directa, es decir, "Niño= Nevada", "Niña= No Nevada".

Son innumerables los artículos sobre el ENSO en todo el Mundo, sin embargo, la mayoría se refieren a su influencia y sus teleconexiones con otros factores ya sean atmosféricos, oceánicos y/o de la cupla océano-atmosfera sobre otras regiones distintas al área bajo estudio. Pero son pocos los que se enfocan en la discriminación precisa de dicha asociación con las nevadas en los Andes Áridos, de las cuales, depende el recurso hídrico que hace posible la existencia de los Oasis Cuyanos y las Áreas Agropecuarias de Chile Central, (Compagnucci, R.H., 2000), Quinn, W H, 1992 Minetti, J. L, W. M. Vargas And Poblete, A.G., 1999. Minetti, J.L, 1984.

Hay una serie de definiciones operativas del ENSO, de las que se desprenden índices para medir y/o estimar su intensidad. En este trabajo se utiliza, el índice Niño 3+4, que mide su componente oceánica en una región específica del Pacífico ecuatorial (Figura 3). Si la anomalía de la temperatura, promediada en dicha área durante tres meses consecutivos, es mayor o igual que $+0,5^{\circ} \mathrm{C}$, se dice que es un evento $\mathrm{El}$ Niño; mientras que, si es menor o igual que $-0,5^{\circ} \mathrm{C}$, se presenta un evento La Niña. Por lo tanto, las condiciones del ENSO se valúan por la magnitud de dicha anomalía y por su duración, (https://climatedataguide.ucar.edu/climate-data/nino-sst-indices-nino-12-3-34-4-oni-and-

tni\#sthash.oD958V9g.dpuf).

En la región de estudio, la asociación entre el ENSO y los derrames de los ríos cuyanos fue tratada por (Campagnucci y Vargas., 1998), (Aceituno y Vidal, 1990), (Aceituno, P. y Garreaud, R., 1995.), (Poblete et. al., 2000, 2013), entre otros; encontrando, en la mayoría de los casos, conexiones directas. Sin embargo, no se ha abordado en profundidad la variabilidad interanual asociada a la de las precipitaciones nivales, teniendo en cuenta la intensidad tanto del evento cálido como la del frío y cuantificado su incidencia.

Investigadores como (Masiokas et. al.2006), (Quintana J.M, 2004), postulan que los factores de gran escala conducen la variabilidad interanual observada, en otras menores, que se manifiestan como cambios rápidos o de alta frecuencia, como es el caso de las precipitaciones en la región central de Chile y Andes adyacentes que, en la mayoría de los mismos, son coherentes con las fases del ENSO (Aceituno P. et. al., 1992). Poblete, encontró una significativa asociación entre los derrames del río San Juan y las SST (Temperaturas Superficiales del Mar), en el área Niño 3+4 (SSTN3+4), (Poblete et. al. 2013)

Este trabajo se propone dilucidar objetivamente y precisar cuantitativamente las aseveraciones respecto de la relación directa entre el ENSO y las nevadas en los Andes Áridos, en el período abrilseptiembre, haciendo uso de herramientas estadísticas específicas y fundamentalmente no paramétricas.

Publicado en formato digital: Dr. Arnobio German Poblete, Ayudante Alumna Maira Antonella Atencio, y Prof. Maria José Vera. CUANTIFICACIÓN DE LA INCIENCIA DEL ENSO EN LAS NEVADAS DE LOS ANDES ÁRIDOS DE SUDAMÉRICA EN EL PERIODO 1909-2015. Revista Geográfica Digital. IGUNNE. Facultad de Humanidades. UNNE. Año 14. N No 27 Enero - Junio 2017. ISSN 1668-5180 Resistencia, Chaco.

En: http://hum.unne.edu.ar/revistas/geoweb/default.htm 
Revista Geográfica Digital. IGUNNE. Facultad de Humanidades. UNNE. Año 14. N²7.

Enero - Junio 2017. ISSN 1668-5180 Resistencia, Chaco

\section{Datos Y Métodos}

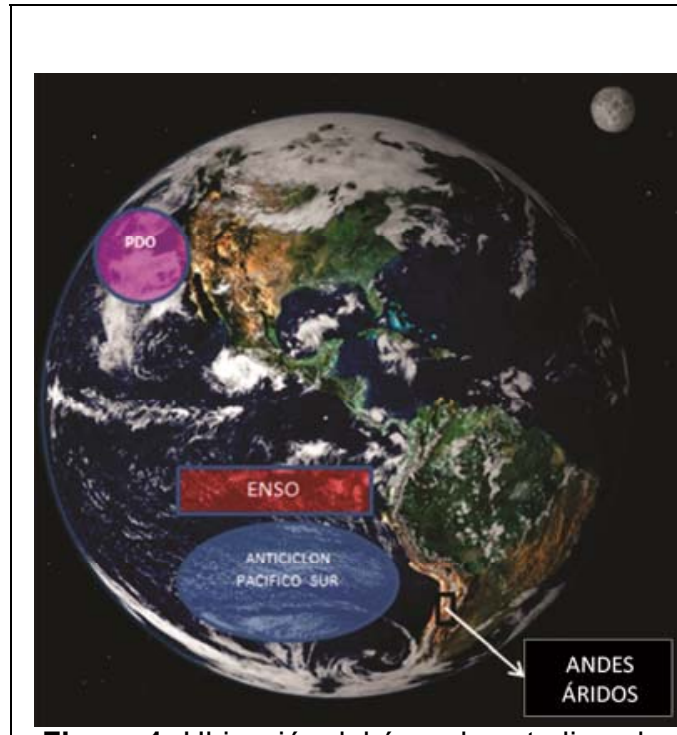

Figura 1: Ubicación del área de estudio y de los factores analizados en este trabajo. Elaboración propia en base a la imagen descargada de http://netflection.blogspot.com.ar/

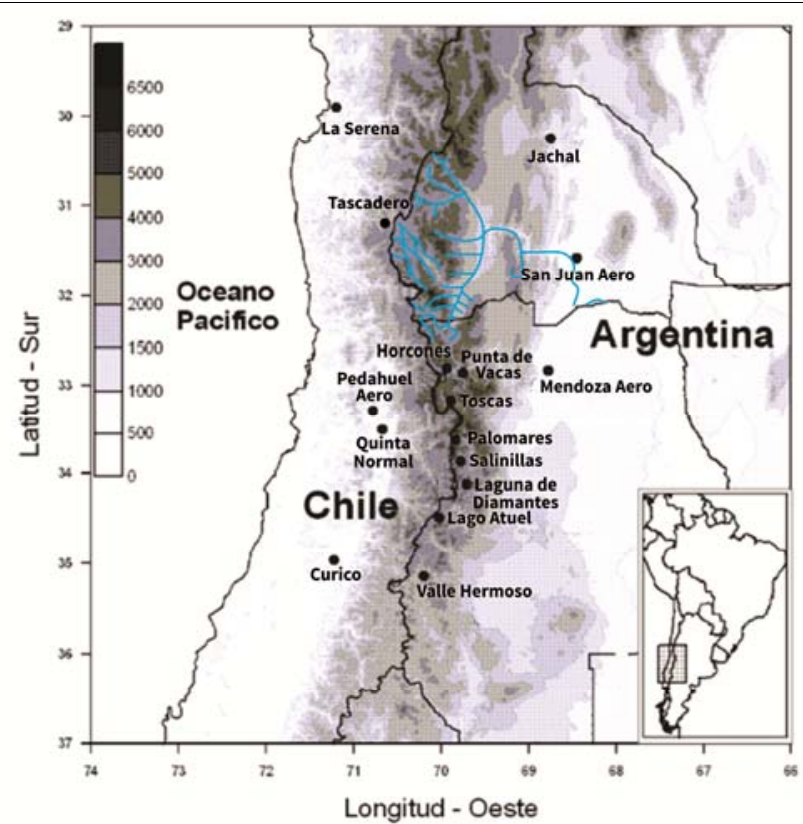

Figura 2: Ubicación de las estaciones de medición de precipitaciones en el área de estudio y del índice que la representa en azul (río San Juan-DJUA-). Adaptado de Viale, (2010).

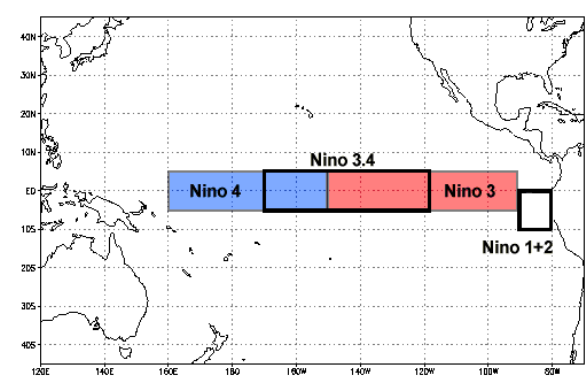

Figura 3: Localización de las Áreas, El Niño. Fuente: http://www.noaa.gov.

Debido a la insuficiencia de mediciones nivales que abarquen un período mayor que 100 años en los Andes Áridos (ver figuras 1), se emplea como índice para estimarlas a los registros de los derrames anuales del río San Juan (DJUA) (ver figuras 2), en el período 1909-2015, puesto que representa lo sucedido en la temporada nival correspondiente (Poblete, 2014). Los datos del DJUA fueron aportados por el Departamento de Hidráulica de la Provincia de San Juan http://www.hidraulica.sanjuan.gov.ar/ y la

Publicado en formato digital: Dr. Arnobio German Poblete, Ayudante Alumna Maira Antonella Atencio, y Prof. Maria José Vera. CUANTIFICACIÓN DE LA INCIENCIA DEL ENSO EN LAS NEVADAS DE LOS ANDES ÁRIDOS DE SUDAMÉRICA EN EL PERIODO 1909-2015. Revista Geográfica Digital. IGUNNE. Facultad de Humanidades. UNNE. Año 14. № 27 Enero - Junio 2017. ISSN 1668-5180 Resistencia, Chaco.

En: http://hum.unne.edu.ar/revistas/geoweb/default.htm 
Revista Geográfica Digital. IGUNNE. Facultad de Humanidades. UNNE. Año 14. N²7.

Enero - Junio 2017. ISSN 1668-5180 Resistencia, Chaco

Secretaría de Recursos Hídricos de la Nación (2014) http://bdhi.hidricosargentina.gov.ar/. Las SSTN3+4 se descargaron del sitio http://www.cpc.ncep.noaa.gov/products.

Los números de las regiones "Niño": 1, 2, 3 y 4 (ver figura 3), se corresponden con los rótulos asignados a las trayectorias de los buques que cruzaban las mismas. Los datos de estas permitieron que los registros históricos de El Niño se retrotrajeran en el tiempo hasta 1949, como se analiza en un estudio clásico de Trenberth, K. (1997).

La región Niño 1+2 (0-10S, 90W-80W), tiene el área más pequeña y oriental de las regiones SST del Niño, es contigua a la región costera de América del Sur, donde El Niño fue reconocido por primera vez por las poblaciones locales. Este índice tiende a tener la mayor variabilidad de todos.

La región Niño 3 (5N-5S, 150W-90W), fue la primer área elegida para el monitoreo y pronóstico de El Niño, pero los investigadores, más tarde, se dieron cuenta que la región clave para valuar las interacciones océano-atmósfera del ENSO se encuentra más al oeste (Trenberth, 1997). Por lo tanto, el Niño 3+4 y el ONI (Oceanic Niño Index, NOAA), se convirtieron en los favoritos para la definición de los eventos cálidos y fríos.

Las anomalías en la región Niño $3+4(5 \mathrm{~N}-5 \mathrm{~S}, 170 \mathrm{~W}-120 \mathrm{~W})$, pueden ser consideradas como el promedio de las SST ecuatoriales del Pacífico, para lo cual, se utilizan normalmente 5 meses significativos del mismo, y el fenómeno de El Niño/La Niña, se define cuando las SSTN3+4 están por encima/debajo de $+0.5^{\circ} \mathrm{C} /-0.5^{\circ} \mathrm{C}$, por un período de seis meses o más. Este es el índice que se utilizará en este artículo.

En la región Niño 4 (5N-5S, 160E-150W): su índice valúa las anomalías de SST en el Pacífico ecuatorial central. Esta área tiende a tener menos variación que las otras.

Las metodologías implementadas fueron:

La distribución Lognormal, que tiene la siguiente función de densidad de probabilidad:

$$
f(x)=\frac{1}{\sigma_{x} \sqrt{2 \pi}} e^{-\frac{1}{2}\left(\frac{\log (x)-\mu}{\sigma_{x}}\right)^{2}}
$$

$\mathrm{e}=$ base de los lg. naturales $=2.71828$

$\pi=3.14159 \ldots \ldots$

$\mu=$ la media de la población

$\sigma_{x}=$ la desviación estandard de la población

$\mathrm{x}=$ una variable. aleatoria contínua $(-\propto \leq \mathrm{x} \leq+\propto)$

Draper N. R., Smith, H. 1998.

\section{Variable Estandarizada}

Mide la desviación de cada dato respecto de la media en unidades de desviación típica.

$$
\text { La nueva variable: } z_{i}=\frac{x_{i}-\bar{X}}{S}
$$

Publicado en formato digital: Dr. Arnobio German Poblete, Ayudante Alumna Maira Antonella Atencio, y Prof. Maria José Vera. CUANTIFICACIÓN DE LA INCIENCIA DEL ENSO EN LAS NEVADAS DE LOS ANDES ÁRIDOS DE SUDAMÉRICA EN EL PERIODO 1909-2015. Revista Geográfica Digital. IGUNNE. Facultad de Humanidades. UNNE. Año 14. N No 27 Enero - Junio 2017. ISSN 1668-5180 Resistencia, Chaco.

En: http://hum.unne.edu.ar/revistas/geoweb/default.htm 
Con $\mathrm{z}_{\mathrm{i}}$ la variable estandarizada obtenida de los $\mathrm{N}$ valores de $\mathrm{x}$ cuya media $(\bar{X})$ y desviación típica $(\mathrm{S})$, tienen una vez tipificadas, una media $=0(\bar{Z}=0)$ y desviación típica $=1\left(S_{z}=1\right)$. Maisel, L. 1973 Otnes, R.K., 1972.

\section{El coeficiente $\tau$ de Kendall.}

Calcula el acuerdo o desacuerdo entre los rangos de dos series temporales y se define como:

Sea $\left(x_{1}, y_{1}\right),\left(x_{2}, y_{2}\right), \ldots,\left(x_{n}, y_{n}\right)$ un conjunto de rangos obtenidos de ordenar las observaciones de las variables originales $X$ e $Y$, respectivamente, de manera que todos los valores de $\left(x_{i}\right)$ e $\left(y_{i}\right)$ son únicos. Cualquier par de observaciones $\left(x_{i}, y_{i}\right)$ y $\left(x_{j}, y_{j}\right)$ se dice que son concordantes:

- Si para $x_{i}>x_{j} y_{i}>y_{j}$ o si $x_{i}<x_{j ;} y_{i}<y_{j}$

- Son discordantes si $x_{i}>x_{j ;} y_{i}<y_{j}$ o si $x_{i}<x_{j}$ y $y_{i}>y_{j}$

- Si $x_{i}=x_{j} \circ y_{i}=y_{j}$, la pareja no es ni concordantes ni discordante.

$$
\tau=\frac{(\text { Nro.acuerdos })-(\text { Nro.desacuerdo } s)}{\frac{n(n-1)}{2}}
$$

El $\tau$ de Kendall también se lo usa como coeficiente de correlación no paramétrico (Kendall 1938). (WMO, 1966).

Si $\tau=1$, hay un total acuerdo, Si $\tau=-1$ total desacuerdo y Si $\tau=0$ no existe ningún tipo de relación. (Siegel, S. y Castellan, N, 1988).

Tablas de Contingencia:

Se utilizan para realizar las pruebas de aleatoriedad para asociaciones categóricas con el correspondiente test ji- cuadrado que tiene la forma, (Siegel, S. y Castellan, N, 1988).

\begin{tabular}{|c|c|c|c|}
\hline CASOS CRITERIO & CRITERIO 1 & CRITERIO 2 & TOTAL \\
\hline CASO 1 & $\mathrm{n}_{11}$ & $\mathrm{n}_{12}$ & $\mathrm{n}_{11}+\mathrm{n}_{12}$ \\
\hline CASO 2 & $\mathrm{n}_{21}$ & $\mathrm{n}_{22}$ & $\mathrm{n}_{21}+\mathrm{n}_{22}$ \\
\hline TOTAL & $\mathrm{n}_{11}+\mathrm{n}_{21}$ & $\mathrm{n}_{12}+\mathrm{n}_{22}$ & $\mathrm{n}_{11}+\mathrm{n}_{21}+\mathrm{n}_{12}+\mathrm{n}_{22}$ \\
\hline
\end{tabular}

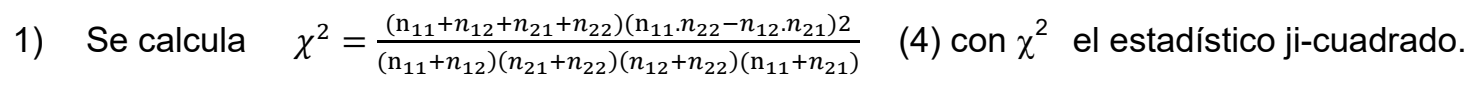

2) Se realiza la siguiente dócima:

$\mathrm{H}_{0}$ : Los casos son independientes.

$\mathrm{H}_{1}$ : No son independientes.

3) Se establece el nivel de confianza $\alpha$ y se busca en la tabla correspondiente el $\chi_{c}^{2}$ para gl grados de libertad.

4) Si $\chi^{2}>\chi_{c}^{2} \Rightarrow$ se rechaza $\mathrm{H}_{0}$

Publicado en formato digital: Dr. Arnobio German Poblete, Ayudante Alumna Maira Antonella Atencio, y Prof. Maria José Vera. CUANTIFICACIÓN DE LA INCIENCIA DEL ENSO EN LAS NEVADAS DE LOS ANDES ÁRIDOS DE SUDAMÉRICA EN EL PERIODO 1909-2015. Revista Geográfica Digital. IGUNNE. Facultad de Humanidades. UNNE. Año 14. N No 27 Enero - Junio 2017. ISSN 1668-5180 Resistencia, Chaco.

En: http://hum.unne.edu.ar/revistas/geoweb/default.htm 
Revista Geográfica Digital. IGUNNE. Facultad de Humanidades. UNNE. Año 14. N²7.

El Coeficiente de Asociación Q de Yule: se define como:

$$
\mathrm{Q}=\frac{\mathrm{n}_{11} \mathrm{n}_{22}-\mathrm{n}_{12} \mathrm{n}_{21}}{\mathrm{n}_{11} \mathrm{n}_{22}+\mathrm{n}_{12} \mathrm{n}_{21}}=\frac{\mathrm{n} \cdot \mathrm{D}}{\mathrm{n}_{11} \mathrm{n}_{22}+\mathrm{n}_{12} \mathrm{n}_{21}}
$$

Cuantifica la asociación entre las variables categóricas que constituyen la tabla de contingencia. Este coeficiente está acotado entre \pm 1 , es decir:

$$
-1 \leq \mathrm{Q} \leq 1
$$

Se interpreta de la siguiente manera:

- Si los atributos $A$ y $B$ son independientes, entonces $Q=0$.

- Si $Q=1$, se verifica que $n_{12} n_{21}=0$ y existe una asociación completa entre los atributos $A$ y $B$.

- Si $Q=-1$, entonces $n_{11} n_{22}=0$ y existe una disociación completa entre $A$ y $B$.

Coeficiente de Contingencia: viene definido por:

$$
C=\sqrt{\frac{X^{2}}{n+X^{2}}}
$$

Si $0 \leq C<1$. Los atributos son independientes cuando $C=0$, mientras que si $C$ está cercano a 1 existe un alto grado de asociación. En el caso de tablas $\mathrm{K} \times \mathrm{K}$ el valor màximo de este coeficiente es $\sqrt{(k-1) / k}$. (Siegel, S. y Castellan, N, 1988).

\section{Resultados Y Discusión}

A continuación se realiza un análisis pormenorizado de la influencia del ENSO sobre las nevadas en los Andes Áridos. Como se dijo, se utilizan los valores del DJUA como índice nival y a la anomalía de la temperatura superficial del mar en el área Niño 3+4 (SSTN3+4), para valuar la componente oceánica del ENSO.

Publicado en formato digital: Dr. Arnobio German Poblete, Ayudante Alumna Maira Antonella Atencio, y Prof. Maria José Vera. CUANTIFICACIÓN DE LA INCIENCIA DEL ENSO EN LAS NEVADAS DE LOS ANDES ÁRIDOS DE SUDAMÉRICA EN EL PERIODO 1909-2015. Revista Geográfica Digital. IGUNNE. Facultad de Humanidades. UNNE. Año 14. NNo 27 Enero - Junio 2017. ISSN 1668-5180 Resistencia, Chaco.

En: http://hum.unne.edu.ar/revistas/geoweb/default.htm 
Revista Geográfica Digital. IGUNNE. Facultad de Humanidades. UNNE. Año 14. №27.

Enero - Junio 2017. ISSN 1668-5180 Resistencia, Chaco

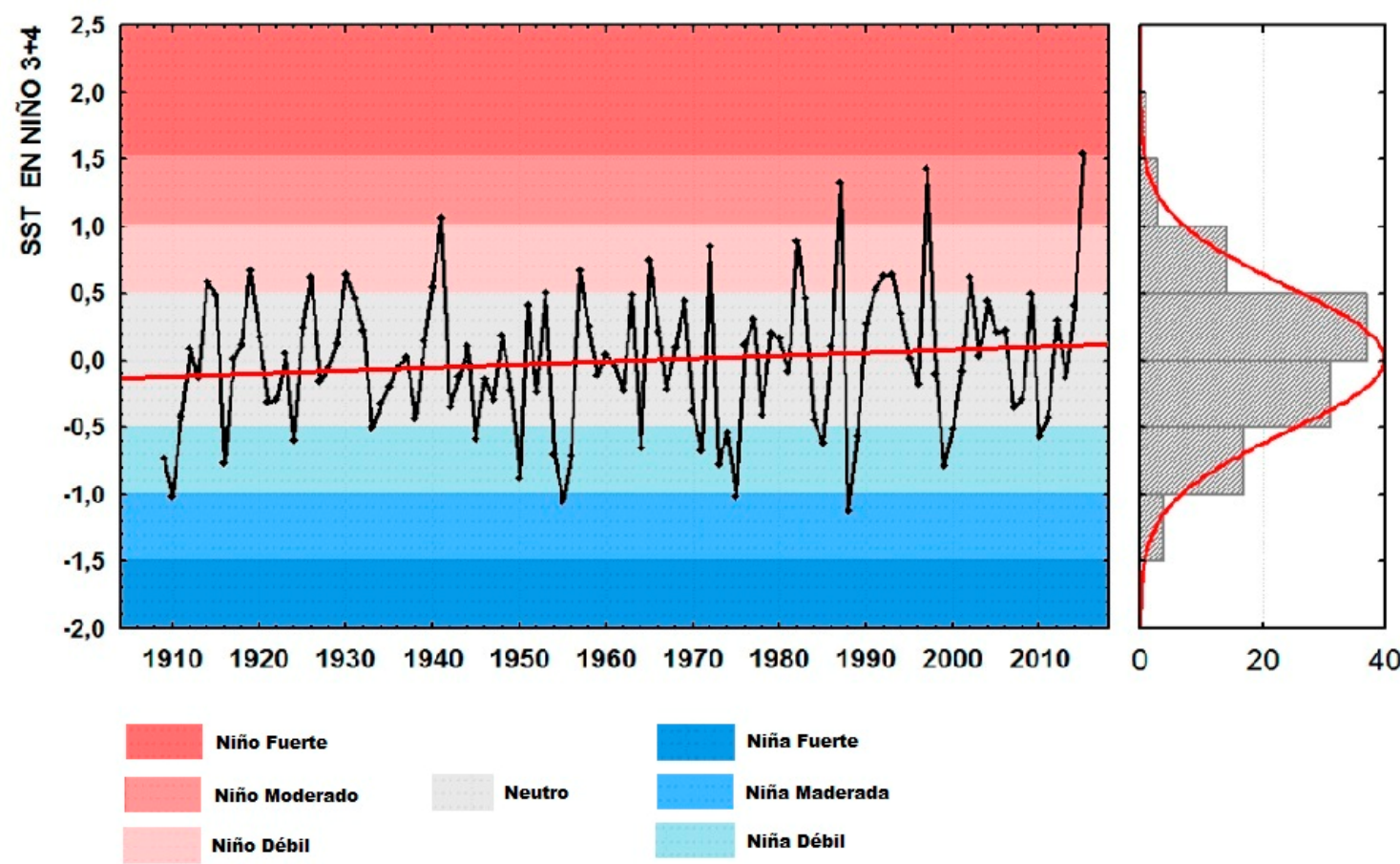

Figura 4: Variabilidad Interanual de las SSTN3+4 en el periodo 1909- 2015, en el lapso abril- septiembre, con su distribución normal y categorías de intensidad. Fuente: Elaboración propia.

En la figura 4, se observa la variabilidad interanual de las SSTN3+4, durante el período nival (abrilseptiembre), presenta una tendencia creciente no significativa y está asociado a una distribución normal. Dominan los eventos Neutros, seguidos por los Fríos y Cálidos débiles, mientras que, solo hay tres Niños y cuatro Niñas Moderados. No se verifican Niños y Niñas en la categoría "Fuerte", con excepción del período 2015. Nótese que ésta no se presenta en muchos casos, debido a que el periodo analizado no incluye los meses cuando el Niño alcanza su climax (en general diciembre). Mientras que, La Niña tiene otra variabilidad estacional.

Publicado en formato digital: Dr. Arnobio German Poblete, Ayudante Alumna Maira Antonella Atencio, y Prof. Maria José Vera. CUANTIFICACIÓN DE LA INCIENCIA DEL ENSO EN LAS NEVADAS DE LOS ANDES ÁRIDOS DE SUDAMÉRICA EN EL PERIODO 1909-2015. Revista Geográfica Digital. IGUNNE. Facultad de Humanidades. UNNE. Año 14. N No 27 Enero - Junio 2017. ISSN 1668-5180 Resistencia, Chaco.

En: http://hum.unne.edu.ar/revistas/geoweb/default.htm 
Revista Geográfica Digital. IGUNNE. Facultad de Humanidades. UNNE. Año 14. N²7.

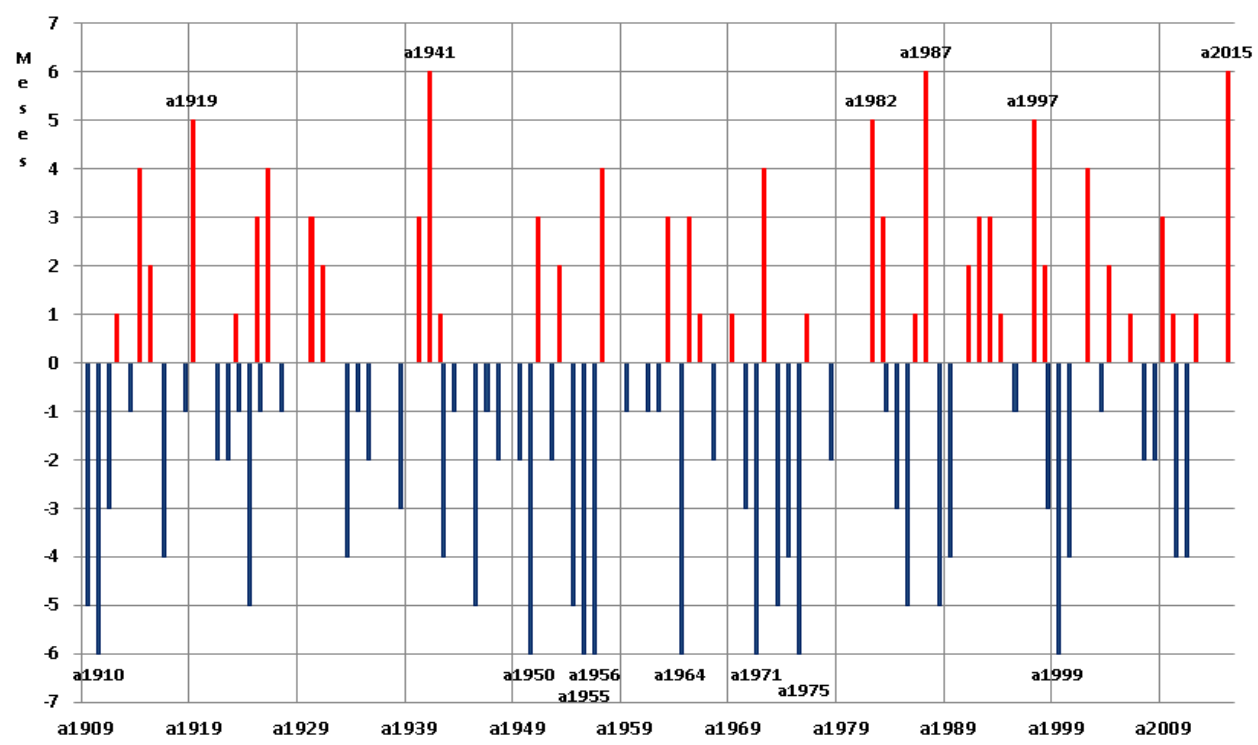

Figura 5: Cantidad de meses en que las SSTN3+4 estuvieron por encima (rojo) y por debajo (azul) de lo normal, durante el período nival (abril- septiembre). Fuente: Elaboración propia.

En el período nival se calcula la cantidad de meses en los que las SSTN3+4 estuvieron por encima o por debajo de la normal. La figura 5, muestra que solo en los años hídricos: 1941-42, 1987-88 y 2015-16, las SSTN3+4 positivas, estuvieron presentes durante los seis meses. Por otra parte, en 1910-11, 1950$51,1955-56,1956-57,1964-65,1971-72,1975-76$ y 1999-2000, se produjeron eventos por debajo de lo normal, con la misma cantidad de meses. De lo que se deduce que los eventos fríos son más persistentes que los cálidos.

Se destaca que los intensos "Niños" de 1982-83 y 1997-98, solo se manifestaron durante cinco meses del periodo nival. Cabe aclarar que en 1919-20 (derrame: $7.085 \mathrm{Hm}^{3}$ ), máximo absoluto de toda la serie, también tuvo cinco meses pero en el marco de un "Niño Débil". A su vez, hubo siete "Niñas" con dicha cantidad de meses, reafirmando lo dicho anteriormente.

Publicado en formato digital: Dr. Arnobio German Poblete, Ayudante Alumna Maira Antonella Atencio, y Prof. Maria José Vera. CUANTIFICACIÓN DE LA INCIENCIA DEL ENSO EN LAS NEVADAS DE LOS ANDES ÁRIDOS DE SUDAMÉRICA EN EL PERIODO 1909-2015. Revista Geográfica Digital. IGUNNE. Facultad de Humanidades. UNNE. Año 14. № 27 Enero - Junio 2017. ISSN 1668-5180 Resistencia, Chaco.

En: http://hum.unne.edu.ar/revistas/geoweb/default.htm 
Revista Geográfica Digital. IGUNNE. Facultad de Humanidades. UNNE. Año 14. N²7.

Enero - Junio 2017. ISSN 1668-5180 Resistencia, Chaco

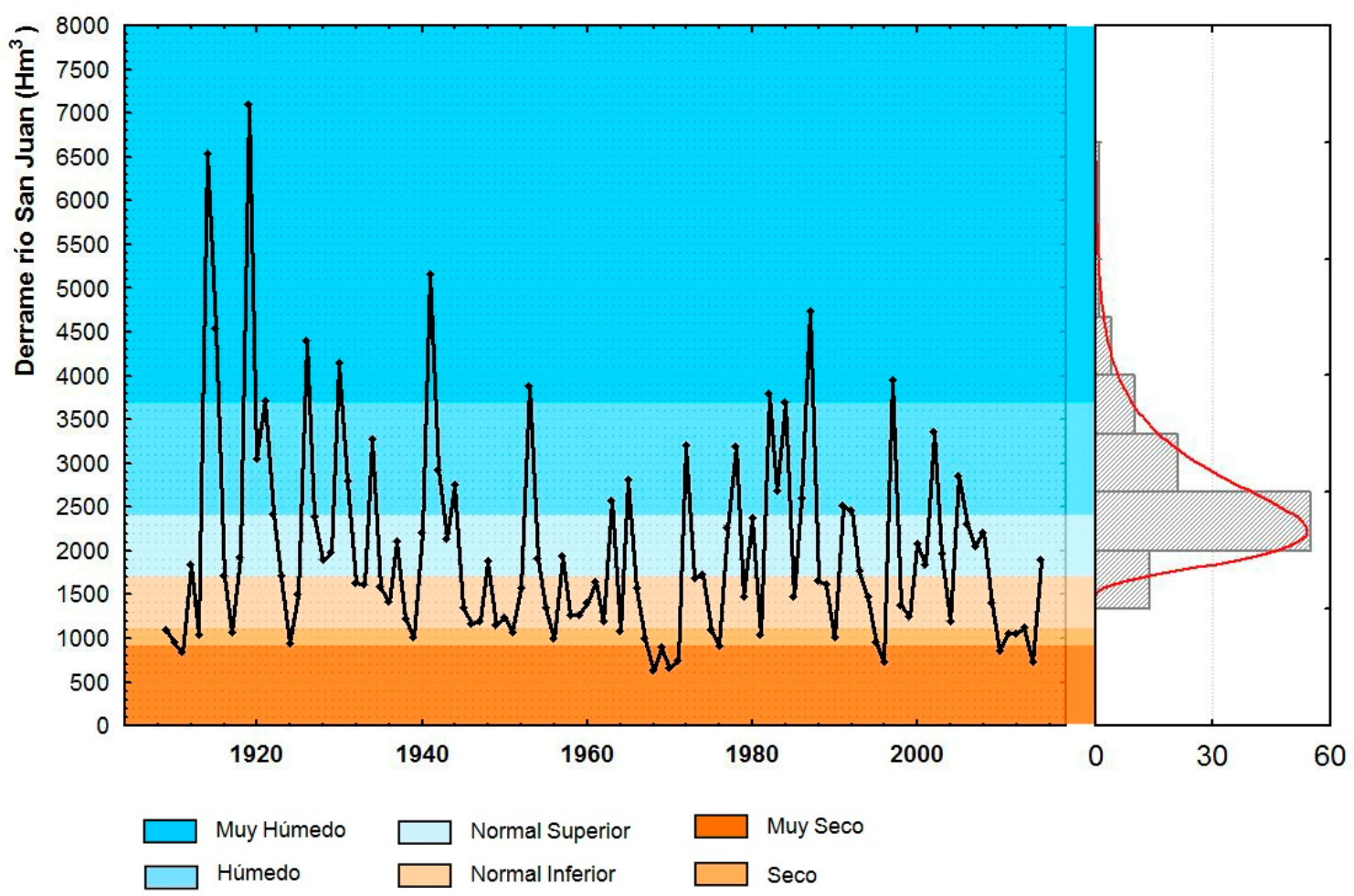

Figura 6: Variabilidad Interanual de los derrames del río San Juan, con su distribución Lognormal y sus niveles de categorización. Fuente: Elaboración propia.

La variabilidad interanual del DJUA se muestra en la figura 6, con su distribución lognormal (tenida en cuenta para determinar el valor promedio de $1784,32 \mathrm{Hm}^{3}$ resultante del $50 \%$ de su frecuencia acumulada, en vez de las corrientemente usadas: media aritmética y mediana) y sus respectivas categorizaciones (ver tabla 1). Obsérvese que las bandas de las mismas tienen diferente amplitud, debido a la distribución citada, de esta manera la más ancha es la "Muy Húmeda", siguiendo su sesgo derecho. En contraste la banda "Seco", resulta muy angosta.

\begin{tabular}{|c|l|}
\hline Muy Húmedo & $\geq$ Decil del $90 \%$ \\
\hline Húmedo & $\geq$ Tercer Cuartil \\
\hline Normal Superior & $\geq 1784,32 \mathrm{Hm}^{3}$ \\
\hline Normal Inferior & $\leq$ Promedio \\
\hline Seco & $\leq$ Primer Cuartil \\
\hline Muy Seco & $\leq$ Decil del $10 \%$ \\
\hline
\end{tabular}

Tabla 1: Categorías asignadas a los derrames anuales del río San Juan de acuerdo a sus deciles y cuartiles.

Publicado en formato digital: Dr. Arnobio German Poblete, Ayudante Alumna Maira Antonella Atencio, y Prof. Maria José Vera. CUANTIFICACIÓN DE LA INCIENCIA DEL ENSO EN LAS NEVADAS DE LOS ANDES ÁRIDOS DE SUDAMÉRICA EN EL PERIODO 1909-2015. Revista Geográfica Digital. IGUNNE. Facultad de Humanidades. UNNE. Año 14. № 27 Enero - Junio 2017. ISSN 1668-5180 Resistencia, Chaco.

En: http://hum.unne.edu.ar/revistas/geoweb/default.htm 
Revista Geográfica Digital. IGUNNE. Facultad de Humanidades. UNNE. Año 14. N²7.

En general, la cantidad de eventos en cada una de las categorías, es la siguiente: Muy Húmedos y Muy Secos son episódicos, esporádicos y escasos; quedando la mayoría concentrados desde la Normal Inferior hasta el Húmedo. Esto se analizará estadísticamente más adelante.

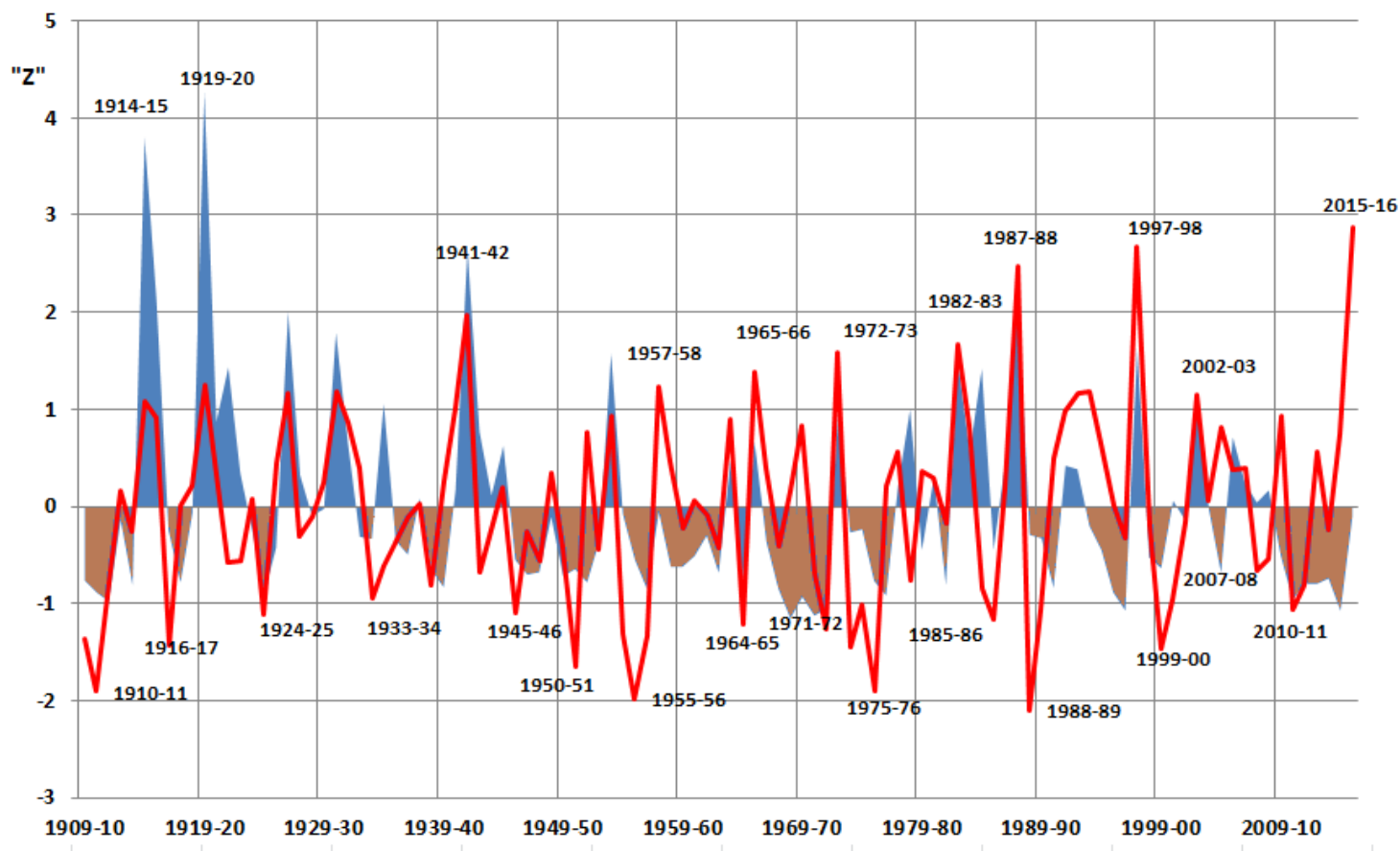

Figura 7: Series temporales estandarizadas de los Derrames del río San Juan (DJUA) (celeste=superávit, marrón= seco) y Anomalías de las Temperaturas Superficiales del Mar (SST) en el área Niño 3+4 (rojo), entre 19092015. Se destacan los periodos con los derrames más relevantes. Fuente: Elaboración propia.

La figura 7 muestra las series temporales estandarizadas del DJUA y de las SSTN3+4, durante el periodo 1909-2015. Se observa que el río San Juan presenta máximos episódicos (azul) de corta duración y gran intensidad, en cambio, los periodos secos (marrón) son de mayor duración y de menor magnitud. Los eventos EI Niño coinciden con los primeros hasta 1941, dicha correspondencia cesa en el periodo comprendido entre 1944 y 1972, donde predominan las sequias, sin embargo, se registran "Niños" importantes tales como: 1957-58 y 1965-66; retomando la coincidencia a partir del 1972-73 hasta el año hídrico 2004-05, cuando se registran los eventos más importantes de toda la serie, tales como 1982-83, 1987-88 y 1997-98, reapareciendo nuevamente en el 2015.

En los períodos secos, se registran varias "Niñas", destacándose las de: 1910-11, 1950-51, 1955-56, 1964-65, 1971-72 (Poblete et. al., 2000) y 1975-76. Reapareciendo en 1988-89, 1999-00 y 2010-11, que inicio la última sequia de importancia que se extendió hasta 2014, puesto que en 2015 domino uno de los Niños más intensos que la revirtió, como se verá más adelante.

Publicado en formato digital: Dr. Arnobio German Poblete, Ayudante Alumna Maira Antonella Atencio, y Prof. Maria José Vera. CUANTIFICACIÓN DE LA INCIENCIA DEL ENSO EN LAS NEVADAS DE LOS ANDES ÁRIDOS DE SUDAMÉRICA EN EL PERIODO 1909-2015. Revista Geográfica Digital. IGUNNE. Facultad de Humanidades. UNNE. Año 14. N No 27 Enero - Junio 2017. ISSN 1668-5180 Resistencia, Chaco.

En: http://hum.unne.edu.ar/revistas/geoweb/default.htm 
Revista Geográfica Digital. IGUNNE. Facultad de Humanidades. UNNE. Año 14. N²7.

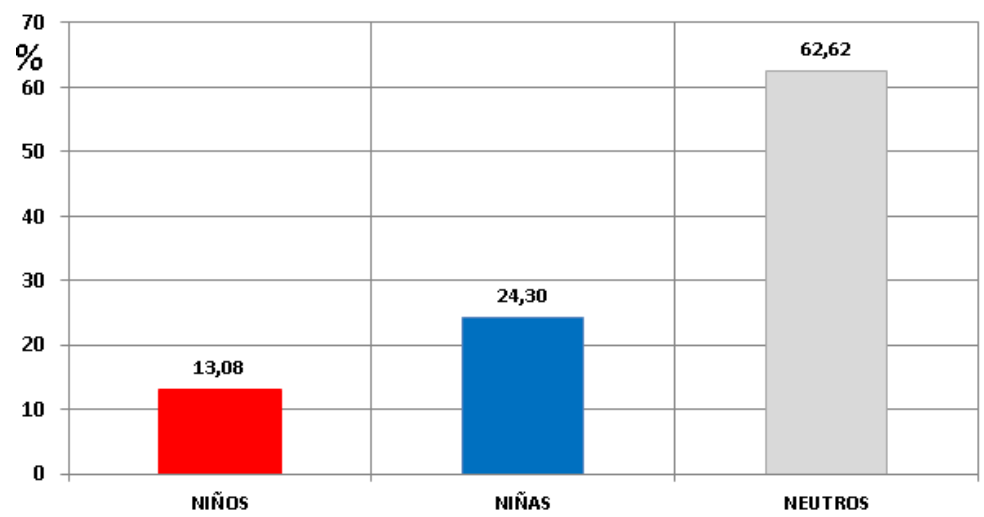

Figura 8: Porcentajes de la ocurrencia de eventos cálidos, fríos y neutros en todo el período 1909-2015. Fuente: Elaboración propia.

En la Figura 8 se visualizan los porcentajes correspondientes a cada evento en todo el período analizado, con un notorio predominio de los Neutros $(62,6 \%)$, seguido de las "Niñas" (24,3\%), y finalmente, los "Niños" (13,8\%), se observa que este ultimo tiene una ocurrencia muy baja, contrariamente a lo que generalmente se cree, puesto que en 106 años solo se presento en 14 durante el periodo abrilseptiembre, mientras que los eventos fríos ocurrieron en 26 años y los Neutros en 66 años.

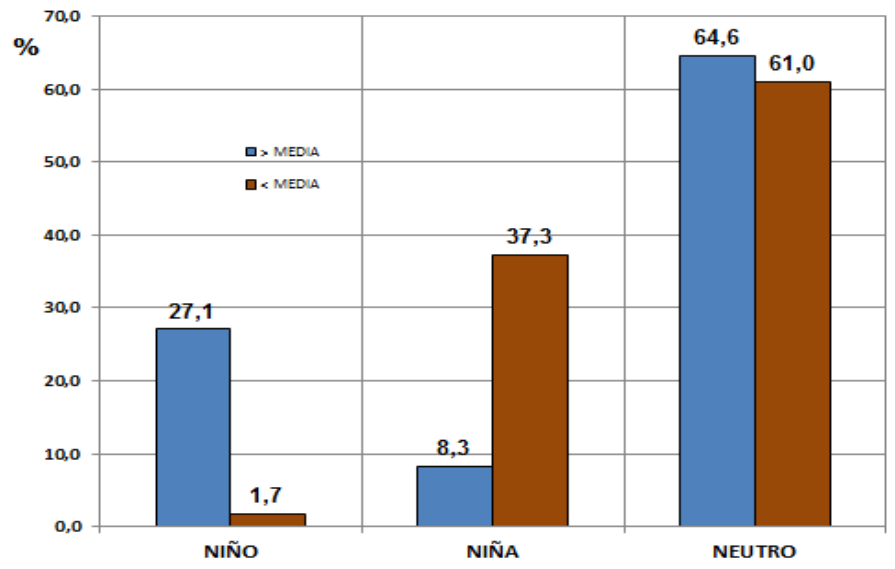

Figura 9: Distribución en porcentajes de los DJUA mayores o menores que su valor medio en función de los eventos Niño, Niña y Neutro. Elaboración propia.

En la figura 9, se observa la relación entre derrames mayores y menores que su valor medio asociados con los eventos cálidos, fríos y neutros del ENSO. Cuando los mismos son superiores que su valor medio hay una ocurrencia del $27,1 \%$ de Niños, $8,3 \%$ de Niñas y $64,6 \%$ de Neutros; mientras que, cuando son menores se presentan $1,7 \%$ Niños, $37,3 \%$ Niñas y $61,0 \%$ Neutros. Lo que muestra un gran predominio de estos últimos en ambos casos. Por otra parte, y en menor medida, prevalecen los "Niños" para derrames mayores y de las "Niñas" en los menores, confirmándose, en este caso, la creencia generalizada pero en porcentajes inferiores a los esperados.

Publicado en formato digital: Dr. Arnobio German Poblete, Ayudante Alumna Maira Antonella Atencio, y Prof. Maria José Vera. CUANTIFICACIÓN DE LA INCIENCIA DEL ENSO EN LAS NEVADAS DE LOS ANDES ÁRIDOS DE SUDAMÉRICA EN EL PERIODO 1909-2015. Revista Geográfica Digital. IGUNNE. Facultad de Humanidades. UNNE. Año 14. № 27 Enero - Junio 2017. ISSN 1668-5180 Resistencia, Chaco.

En: http://hum.unne.edu.ar/revistas/geoweb/default.htm 
Revista Geográfica Digital. IGUNNE. Facultad de Humanidades. UNNE. Año 14. N²7.

\begin{tabular}{|c|c|c|c|}
\hline & NIÑO & NIÑA & NEUTRO \\
\hline$>\mathrm{ME}$ & 13 & 4 & 31 \\
\hline$<\mathrm{ME}$ & 1 & 22 & 36 \\
\hline \multicolumn{4}{|c|}{$\begin{array}{l}\text { Yates Corrección }=0,5 . \mathbf{X}^{2}=19,16162 . \mathbf{p} \text { - } \\
\text { value }=0,00007 . \mathbf{Q} \text { de Yule }=\text { table is not } 2 \times 2 \text {. } \\
\text { Pearson } \mathbf{C}=0,38972 \text {. Corregido } \mathbf{C}=\text { number } \\
\text { of rows and columns is not equal. Cramer } \mathbf{V}= \\
0,42318 . \mathbf{m}=1 . \\
\text { Tabla } 2\end{array}$} \\
\hline
\end{tabular}

\begin{tabular}{|c|c|c|}
\hline & NIÑO & NO NIÑO \\
\hline$>\mathrm{ME}$ & 13 & 35 \\
\hline$<\mathrm{ME}$ & 1 & 58 \\
\hline \multicolumn{3}{|c|}{ 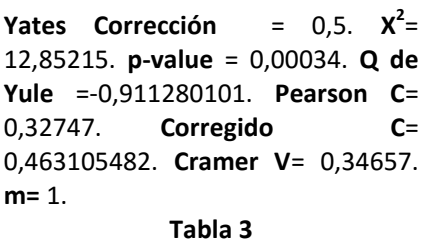 } \\
\hline
\end{tabular}

\begin{tabular}{|c|c|c|}
\hline & NIÑA & NO NIÑA \\
\hline$>\mathrm{ME}$ & 4 & 35 \\
\hline$<\mathrm{ME}$ & 22 & 58 \\
\hline \multicolumn{3}{|c|}{$\begin{array}{l}\text { Yates Corrección }=0,5 . \mathbf{X}^{2}=3,6116 . \mathrm{p}- \\
\text { value }=0,0538 . \text { Q de Yule }= \\
0,536926148 . \text { Pearson } \mathrm{C}=0,17163 . \\
\text { Corregido } \mathrm{C}=0,242716439 . \text { Cramer V- } \\
0,17421 . \mathrm{m}=1 . \\
\text { Tabla 4 }\end{array}$} \\
\hline
\end{tabular}

Para valuar la significación de lo mostrado en el párrafo anterior se implementa un análisis estadístico, haciendo uso de tablas de contingencias (ecuación 4). La Tabla 2 evalúa la asociación entre todos los eventos posibles en relación a los derrames mayor/menor que su promedio. Confirmándose dicha asociación puesto que $X^{2}$ es significativo al $1 \%$ (valor de $p=0,00007)$. Es decir, que hay una correspondencia entre la ocurrencia de los eventos con el hecho que el derrame sea mayor o menor que su media.

En la Tabla 3 se muestra el análisis de la ocurrencia de un evento "Niño" vs. "No Niño", siendo la asociación significativa al $1 \%$ (valor de $p=0,00034$ ) y un $Q$ de Yule $=-0,911280101$, confirmando la alta asociación encontrada con el $\mathrm{X}^{2}$.

Se observa en la Tabla 4, la asociación entre la "Niña" vs. "No Niña", dando una significación notoriamente menor al $5 \%$ y un $Q$ de Yule $=-0,54$, es decir, inversa. Diferenciándose del resto por el escaso peso que tienen las "Niñas" presentes en los derrames mayores que la media.

\begin{tabular}{|c|c|c|}
\hline & NIÑO & NEUTRO \\
\hline$>$ ME & $\mathbf{1 3}$ & $\mathbf{3 1}$ \\
\hline$<$ ME & $\mathbf{1}$ & $\mathbf{3 6}$ \\
\hline \multicolumn{3}{|c|}{ Yates Corrección $=0,5 . \mathbf{X}^{2}=8,33900$. p-value } \\
$=0,00388$. Q de Yule $=0,875751503$. Pearson \\
C= 0,30552. Corregido C= 0,432067427. \\
Cramer V=0,320886. $\mathbf{m}=1$. \\
Tabla $\mathbf{5}$
\end{tabular}

\begin{tabular}{|c|c|c|}
\hline & NIÑA & NEUTRO \\
\hline$>\mathrm{ME}$ & 4 & 31 \\
\hline$<M E$ & 22 & 36 \\
\hline \multicolumn{3}{|c|}{$\begin{array}{c}\text { Yates Corrección }=0,5 . \mathbf{X}^{2}=6,35310 \\
\text { p-value }=0,01172 . \text { Q de Yule }= \\
0,651331719 . \text { Pearson } \mathbf{C}=0,25287 \\
\text { Corregido } \mathbf{C}=0,357616201 . \text { Cramer } \\
\text { V=0,26137. } \mathbf{m}=1 . \\
\text { Tabla } 6\end{array}$} \\
\hline
\end{tabular}

\begin{tabular}{|c|c|c|}
\hline & NIÑO & NIÑA \\
\hline$>M E$ & 13 & 4 \\
\hline$<\mathrm{ME}$ & 1 & 22 \\
\hline \multicolumn{3}{|c|}{$\begin{array}{l}\text { Yates Corrección }=0,5 . \mathbf{X}^{2}=19,29232 . \mathbf{p}- \\
\text { value }=0,00001 . \text { Q de Yule }= \\
0,972413793 . \text { Pearson C= } 0,57042 . \\
\text { Corregido C }=0,806692457 . \text { Cramer V= } \\
\text { 0,69448. } \mathbf{m}=1 . \\
\text { Tabla 7 }\end{array}$} \\
\hline
\end{tabular}

En la Tabla 5, se estima la asociación "Niño" vs. Neutros, se verifica que tanto el $X^{2}$ como el $Q$ de Yule son altamente significativos, confirmando lo determinante que resultan los derrames de ser mayores/menores que su promedio respecto de la ocurrencia de eventos cálidos/neutros.

La asociación entre "Niña" y Neutros se estima en la Tabla 6, al igual que cuando se la comparo con la "No Niña" la asociación es inversa y con una significación menor por la misma razón allí expuesta.

Por último, se analiza la asociación entre "Niño" vs. "Niña" en relación a que el derrame sea mayor o menor que su promedio. La Tabla 7, muestra los resultados del mismo, verificándose una significativa relación corroborada por un $\mathrm{X}^{2}$ y un $\mathrm{Q}$ de Yule $=0,972413793$, significativo ambos al $1 \%$ o. Poniendo de manifiesto el primero, la mayor asociación entre los contrates analizados.

Publicado en formato digital: Dr. Arnobio German Poblete, Ayudante Alumna Maira Antonella Atencio, y Prof. Maria José Vera. CUANTIFICACIÓN DE LA INCIENCIA DEL ENSO EN LAS NEVADAS DE LOS ANDES ÁRIDOS DE SUDAMÉRICA EN EL PERIODO 1909-2015. Revista Geográfica Digital. IGUNNE. Facultad de Humanidades. UNNE. Año 14. N No 27 Enero - Junio 2017. ISSN 1668-5180 Resistencia, Chaco.

En: http://hum.unne.edu.ar/revistas/geoweb/default.htm 
Revista Geográfica Digital. IGUNNE. Facultad de Humanidades. UNNE. Año 14. N²7.

\section{Análisis de Extremos}

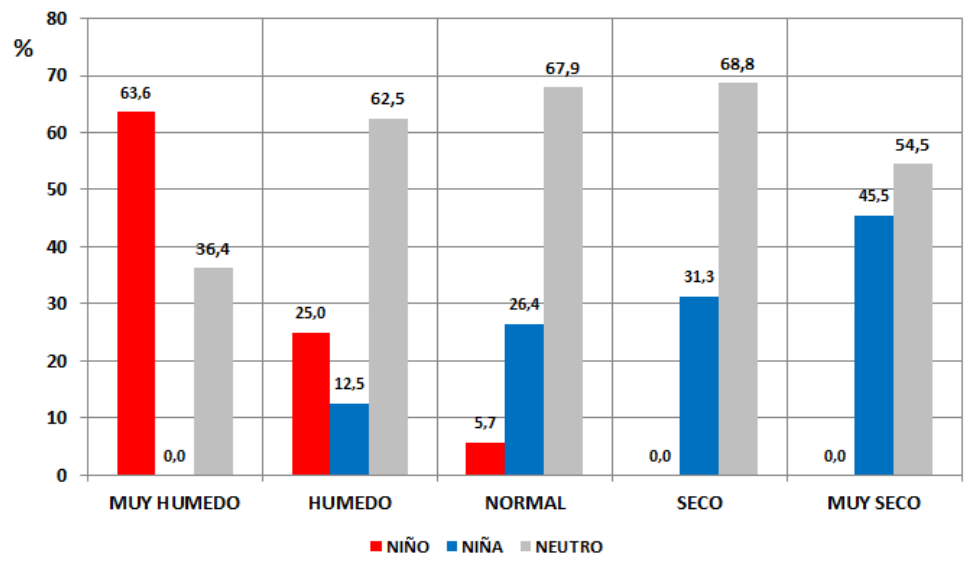

Figura 10: Ocurrencia de eventos Niño, Niña y Neutro en porcentajes de acuerdo a la categoría de los DJUA (ver figura 6). Fuente: Elaboración propia.

Teniendo en cuenta la categorización de los derrames mostrada en la figura 6 , se analiza la ocurrencia de los eventos Cálidos, Fríos y Neutros, en cada una de ellas. En la figura 10, se observa que en los Muy Húmedos, predominan los "Niños" con el $63,6 \%$, siguiéndole los Neutros con $36,4 \%$ y Niñas ausentes. En los Húmedos, la presencia del Niño se reduce a $25 \%$, mientras que se registran los eventos fríos con un 12,5\%, dominando los Neutros con un 62,5\%. En los derrames Normales dominan los Neutros con un $67,9 \%$, siguiéndole las Niñas con un $26,4 \%$, disminuyendo los Niños a un $5,7 \%$. En la categoría Seco, también tiene un gran predominio de los Neutros (68,8\%), luego las Niñas con $31,3 \%$, sin presencia de los "Niños". Por último, en los Muy Secos, dominan los Neutros con el $54,5 \%$, le siguen las "Niñas" con el $45,5 \%$, confirmándose de esta manera la asociación entre éstas con los derrames muy bajos además de la ausencia de Niños.

Lo descripto corrobora que los eventos cálidos se asocian a derrames de "Altos" a "Muy Altos", mientras que Las Niñas en los "Secos" a "Muy Secos", pero en menor proporción. Todo en un marco con predominio de Neutros. Es notable también la inexistencia de Niñas/Niños en los categorías "Muy Húmedos"/“Muy Seco"-“Seco". Sin embargo, se verifica un pequeño porcentaje de "Niñas" en la categoría Húmedo.

\section{Conclusiones}

La variabilidad interanual de las SSTN3+4, durante el período nival (abril- septiembre), tienen una tendencia creciente no significativa y están asociadas a una distribución normal. En base a su categorización, se verifica un notorio predominio de los Neutros $(62,6 \%)$, seguido por las "Niñas" $(24,3 \%)$, y finalmente, los "Niños" con una ocurrencia muy baja $(13,8 \%)$, contrariamente a lo que generalmente se cree, puesto que en 106 años, este ultimo solo se presento en 14, mientras que los eventos fríos ocurrieron en 26 y los neutros en 66 años.

Publicado en formato digital: Dr. Arnobio German Poblete, Ayudante Alumna Maira Antonella Atencio, y Prof. Maria José Vera. CUANTIFICACIÓN DE LA INCIENCIA DEL ENSO EN LAS NEVADAS DE LOS ANDES ÁRIDOS DE SUDAMÉRICA EN EL PERIODO 1909-2015. Revista Geográfica Digital. IGUNNE. Facultad de Humanidades. UNNE. Año 14. N No 27 Enero - Junio 2017. ISSN 1668-5180 Resistencia, Chaco.

En: http://hum.unne.edu.ar/revistas/geoweb/default.htm 
Revista Geográfica Digital. IGUNNE. Facultad de Humanidades. UNNE. Año 14. №27.

Enero - Junio 2017. ISSN 1668-5180 Resistencia, Chaco

Por la cantidad de meses en los que las SSTN3+4 estuvieron por encima o por debajo de lo normal, se verifico que los eventos fríos son más persistentes que los cálidos. Los intensos "Niños" de 1982-83 y 1997-98, se manifestaron durante solo cinco meses del periodo nival y en 1919-20, se produjeron cinco meses pero en el marco de un "Niño" débil, sin embargo, en ese periodo se produjo un derrame de 7.085 $\mathrm{Hm}^{3}$, el máximo absoluto de toda la serie.

De la categorización de la variabilidad interanual del DJUA, se comprobó que la cantidad de eventos en cada una de ellas, es la siguiente: Muy Húmedos y Muy Secos son episódicos, esporádicos y escasos; quedando la mayoría concentrados desde la Normal Inferior hasta el Húmedo.

En las series temporales estandarizadas del DJUA y de las SSTN3+4, se evidenció que el río San Juan presenta máximos episódicos de corta duración y gran intensidad, en cambio, los periodos secos son de mayor persistencia y menor magnitud.

Con la aplicación de las tablas de contingencia se comprobó que la asociación entre el ENSO vs. Derrames Mayores/Menores al valor medio, es significativa de acuerdo a los test $\mathbf{X}^{2}$ y $Q$ de Yule.

Se corroboró que los eventos cálidos se asocian a derrames desde "Altos" a "Muy Altos", mientras que los fríos desde los "Secos" a "Muy Secos", en menor proporción. Todo en un marco con predominio de Neutros. Es notable también la inexistencia de Niñas/Niños en las categorías "Muy Húmedos" / "Muy Seco"-“Seco". Presentándose un pequeño porcentaje de "Niñas" en la categoría Húmedo.

En particular, el 2015 (derrame: $1.985 \mathrm{Hm}^{3}$ ), fue uno de los "Niños" con una categorización semejante a los intensos eventos cálidos de 1941-42 $\left(5.152 \mathrm{Hm}^{3}\right), 1982-83\left(3.793 \mathrm{Hm}^{3}\right), 1987-88\left(4.664 \mathrm{Hm}^{3}\right), y$ 1997-98 (3.935 $\left.\mathrm{Hm}^{3}\right)$, sin embargo, no alcanzó los derrames de aquellos, solo superando mínimamente su promedio.

Por todo lo expuesto, los autores infieren que el ENSO funcionaría solo como un disparador de los otros factores que inciden en la caída de nieve, estando nítidamente asociados los eventos Cálidos con derrames muy altos y en menor medida los Fríos con los muy bajos, de ahí que la creencia generalizada solo se verifica en los extremos. Es significativo el notable predominio de los Neutros en todos los casos.

\section{Agradecimientos}

Se agradece a la Universidad Nacional de San Juan, por sus aportes económicos en el desarrollo de este trabajo y "Análisis de las Condiciones de Borde, Factores y Agentes Oceánicos de Circulación Atmosférica Regional y de la Cupla Océano Atmósfera que Inciden en las Nevadas de Los Andes Áridos". Res. 1531-R-16/CS-UNSJ.

\section{Bibliografía}

$\checkmark$ Aceituno, P. Y Garreaud, R., 1995. Impactos de los fenómenos El Niño y La Niña sobre regímenes fluviométricos andinos. Rev. Soc. Chilena Ing. Hidráulica, vol. 10(2), pp. 33-43.

$\checkmark$ Aceituno, P. Y Vidal, F. 1990. Variabilidad interanual en el caudal de ríos andinos en Chile Central en relación con la temperatura de la superficie del mar en el Pacífico Central. Revista de la Sociedad Ingeniería Hidráulica 5:1, n.p.

$\checkmark$ Aceituno, P.; Fuenzalida, H. and Rosenbluth, B. (1992): "Climate along the extratropical West coast of South America", in H. A. Mooney; B. Kronberg and E. R. Fuentes (eds.): Earth Systems responses to global change. Michigan, Academic Press, pp. 61-69.

Publicado en formato digital: Dr. Arnobio German Poblete, Ayudante Alumna Maira Antonella Atencio, y Prof. Maria José Vera. CUANTIFICACIÓN DE LA INCIENCIA DEL ENSO EN LAS NEVADAS DE LOS ANDES ÁRIDOS DE SUDAMÉRICA EN EL PERIODO 1909-2015. Revista Geográfica Digital. IGUNNE. Facultad de Humanidades. UNNE. Año 14. N No 27 Enero - Junio 2017. ISSN 1668-5180 Resistencia, Chaco.

En: http://hum.unne.edu.ar/revistas/geoweb/default.htm 
Revista Geográfica Digital. IGUNNE. Facultad de Humanidades. UNNE. Año 14. №27.

Enero - Junio 2017. ISSN 1668-5180 Resistencia, Chaco

$\checkmark$ COMPAGNUCCI, R.H. Y VARGAS, W.M., 1998. Interannual variability of Cuyo Rivers Streamflow in Argentinean Andean Mountains and ENSO events. International Journal of Climatology, vol.18, pp. 1593-1609.

$\checkmark$ Compagnucci, R.H., 2000. ENSO events impact on hydrological system in the Cordillera de los Andes during the last $\mathbf{4 5 0}$ years. En el libro: Southern Hemisphere Paleo-and Neoclimates: Methods and Concepts. Eds. Volkheimer W. and P. Smolka. Editorial Springer Verlag, pp. 175-185.

$\checkmark$ Draper Norman R., Smith, Harry. 1998. Applied Regression Analysis, 3rd Edition. Ed. Wiley. ISBN: 978-0-471-17082-2. 736 pages. New York.

$\checkmark$ Kiladis, G. N.And H.F. Diaz, 1989: Global Climatic Anomalies Associated with Extremes in the Southern Oscillation. J. of Climate 2, 1069-1089.

$\checkmark$ Maisel, L. 1973. Probabilidad y Estadística. Fondo educativo interamericano. México.

$\checkmark$ Masiokas, M.H., et al. 2006. "Snowpack Variations in the Central Andes of Argentina and Chile, 19512005: Large-Scale Atmospheric Influences and Implications for Water Resources in the Region". JOURNAL OF CLIMATE VOLUME 19. American Meteorological Society. New York.

$\checkmark$ Minetti, J. L, W. M. Vargas and Poblete A.G., 1999. Monitoring El "Niño"/ La "Niña" Event Through the Temperature of Lima (Peru).Climatic Change.

$\checkmark$ Minetti, J.L, 1984. Algunos Avances En Métodos Predictivos Para El Escurrimiento Superficial De Ríos Andinos Centrales en la República Argentina. Jornadas de Hidrología de nieves y hielos en América del Sur. Programa Hidrológico Internacional UNESCO. Santiago-Chile.

$\checkmark$ NOAA 2015, NACIONAL OCEANIC AND ATMOSPHERIC ADMINISTRATION, http://www.noaa.gov/.

$\checkmark$ Otnes, R.K., 1972. Digital time series analysis. John Wiley \& Sons, 467 pp.

$\checkmark$ Poblete, A. G. y Sánchez G. 2000. "Análisis de la posible asociación entre los derrames anuales del río San Juan y el fenómeno de "EI niño" /"La niña". Revista de Geografía No: 4 del Instituto y Departamento de Geografía de la UNSJ. ISSN 1544-1942. Pg. 27-36.

$\checkmark$ Poblete A. G., Minetti J.L., Iranzo D. A. 2013. "Condiciones de Borde Atmosféricas y Oceánicas que Intervienen en la Alimentación Nívea de los ríos Cuyanos". San Juan.

$\checkmark$ Poblete Arnobio. Minetti, Juan L. Aguiar L.A. 2014. Distribución Espacial Del Recurso Hídrico Superficial De La Provincia De San Juan. Revista de Geografía • Número 18 • Año 2014 • Vol. XVII • ISSN 1514-1942 • San Juan - Argentina.

$\checkmark$ QUINN,W H, 1992 A study of the Southern Oscillation- related climatic activity for A.D.622-1900 incorporating Nile River flood data. El "Niño"- Historical and paleoclimatic aspects of the Southern Oscillation Chapter Six, 121- 149.

$\checkmark$ Quintana, J. M., 2004: Factors affecting Central Chile rainfall variations at interdecadal scales (in Spanish). M.S. thesis, Departamento de Geofísica, Universidad de Chile, 88 pp.

$\checkmark$ SIEGEL, S., CASTELLAN, N. "Nonparametric Statistics for the Behavioir”. Sciene. Ed. Trillas. Madrid.1988.

$\checkmark$ TRENBERTH, K. 1997. The definition of El "Niño". National Center for Atmospheric Research. Boulder. Colorado. USA.

$\checkmark$ Viale, Maximiliano, 2010. Características de las Precipitaciones Orográficas de invierno sobre los Andes Subtropicales Centrales. Universidad de Buenos Aires. Facultad de Ciencias Exactas y Naturales. Departamento de Ciencias de la Atmósfera y los Océanos.

Publicado en formato digital: Dr. Arnobio German Poblete, Ayudante Alumna Maira Antonella Atencio, y Prof. Maria José Vera. CUANTIFICACIÓN DE LA INCIENCIA DEL ENSO EN LAS NEVADAS DE LOS ANDES ÁRIDOS DE SUDAMÉRICA EN EL PERIODO 1909-2015. Revista Geográfica Digital. IGUNNE. Facultad de Humanidades. UNNE. Año 14. N No 27 Enero - Junio 2017. ISSN 1668-5180 Resistencia, Chaco.

En: http://hum.unne.edu.ar/revistas/geoweb/default.htm 